\title{
Tevatron Searches for Excited and Exotic Leptons
}

\author{
S. Hagopian \\ on behalf of the $\mathrm{D} \varnothing$ and CDF Collaborations \\ Florida State University - Department of Physics \\ Tallahassee, Florida - U.S.A.
}

\begin{abstract}
The Tevatron experiments, $\mathrm{D} \varnothing$ and $\mathrm{CDF}$, have searched for excited electrons and excited and exotic muons in Run II. Using $1 \mathrm{fb}^{-1}$ of data, D $\varnothing$ has searched for excited electrons. No excess above the standard model background is observed. Choosing the scale for contact interactions to be $\Lambda=1 \mathrm{TeV}$, excited electron masses below $756 \mathrm{GeV}$ are excluded at the $95 \%$ C.L. CDF has searched for excited and exotic muons using $371 \mathrm{fb}^{-1}$ of data. Using gauge mediated models with $\Lambda / \mathrm{f}=m_{\mu^{*}}$, exotic muons are excluded for masses below $221 \mathrm{GeV}$. For compositeness models with $\Lambda=m_{\mu^{*}}$, masses below $853 \mathrm{GeV}$ are excluded. Using a similar size data set, DØ get similar limits.
\end{abstract}

\section{Introduction}

The proliferation of quarks and leptons and their mass hierarchy motivates composite models, where the quarks and leptons are composed of scalar and spin $1 / 2$ particles leading to a spectrum of excited states $\mathrm{e}^{*}, \mu^{*}$, and $\mathrm{q}^{*}[2]$. Their production can be described by contact interactions (CI) between quarks and leptons. Their decays can be via electroweak interactions or via contact interactions[3]. Exotic fermions with a spectrum of excited states are also predicted by extensions of the standard model such as gauge-mediated models (GM)[4].

\section{DØ Search for Excited Electrons}

$\mathrm{D} \varnothing$ searches for $e^{*}$ in the process $p \bar{p} \rightarrow e^{*} e$, with the $e^{*}$ subsequently decaying to an electron plus photon. Using $1 \mathrm{fb}^{-1}$ of data, D $\varnothing$ has selected two isolated electrons with high transverse energy $\left(E_{T}\right)$ and one isolated high $E_{T}$ photon, resulting in 259 events with an estimated standard model background of $232 \pm 36$ events. The background is dominated by the Drell-Yan process, DY $+\gamma \rightarrow e^{+} e^{-} \gamma$. Since no excess is seen, D $\varnothing$ calculates $95 \%$ C.L. limits. The resulting limit as a function of $m_{e^{*}}$ is shown in Fig. 1 together with predictions of the contact interaction model for different choices of the scale $\Lambda$. The effect of both CI and GM decays is included, unless otherwise noted. Previous limits obtained by CDF are also shown[5]. For $\Lambda=1 \mathrm{TeV}\left(\Lambda=m_{e^{*}}\right)$, masses below $756 \mathrm{GeV}(796 \mathrm{GeV})$ are excluded. Reference [6] has detailed information on this analysis.

\section{CDF Search for Excited and Exotic Muons}

CDF has searched for excited and exotic muons in $371 \mathrm{fb}^{-1}$ of data. After selecting two isolated high $E_{T}$ muons and one isolated high $E_{T}$ photon, 17 events remain, with a background of $8.7 \pm 0.9$ events. The dominant background is $\mathrm{Z} \gamma$. The data excess is predominantly in the $\mathrm{Z} \rightarrow \mu \mu \gamma$ final state radiation region, with 5.5 events predicted and 11 candidate events observed. The resulting limit on cross section times branching fraction as a function of $m_{\mu^{*}}$ is shown in Fig. 2 together with predictions of the contact interaction and gauge-mediated

DIS 2008 


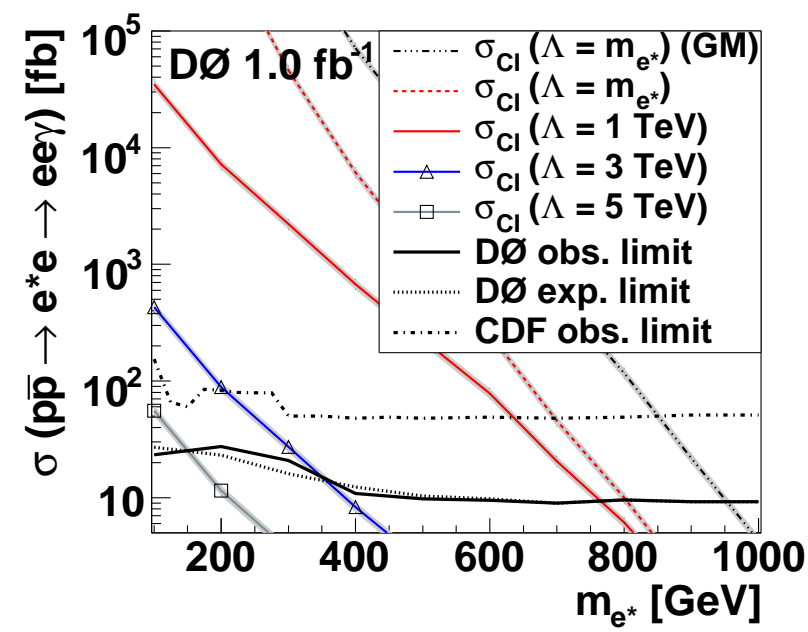

Figure 1: The DØ measured and expected limits on cross section times branching fraction, compared to the contact interaction model prediction for different choices of $\Lambda$. Also shown is the prediction under the assumption that no decays via contact interactions occur ("GM"), and the CDF result [5]. The theoretical uncertainty of the model prediction is indicated by shaded bands.

models for different choices of parameters. Using a gauge mediated model with $\Lambda / \mathrm{f}=m_{\mu^{*}}$, (f/ $\left.\Lambda=10^{-2} \mathrm{GeV}\right)$, where $\mathrm{f}$ is the gauge coupling factor, exotic muons are excluded for masses below $221 \mathrm{GeV}(410 \mathrm{GeV})$. For compositeness models with only electroweak decays and $\Lambda=m_{\mu^{*}}$, masses below $853 \mathrm{GeV}$ are excluded. Details and 2-D exclusion regions are given in reference [7].

\section{DØ Search for Excited Muons}

DØ has searched for excited muons in $380 \mathrm{fb}^{-1}$ of data. The selection of at least one isolated high $E_{T}$ muon, a second high $E_{T}$ muon and one isolated high $E_{T}$ photon, yields 90 events with a background of $65 \pm 40$ events. The dominant background is $\mathrm{Z} \gamma$. The large uncertainty in the background estimate is due to misidentified photons, which are negligible for large $m_{\mu^{*}}$. The resulting limits on cross section times branching fraction, including the effect of CI decays, is shown in Fig. 3 as a function of $m_{\mu^{*}}$ together with predictions of the contact interaction model for different choices of the scale $\Lambda$. For $\Lambda=1 \mathrm{TeV}\left(\Lambda=m_{\mu^{*}}\right)$, masses below $618 \mathrm{GeV}(688 \mathrm{GeV})$ are excluded. For assumptions similar to CDF, $m_{\mu^{*}}$ values below $890 \mathrm{GeV}$ are excluded. More information is available in reference [8].

\section{Summary}

DØ and CDF have searched for excited electrons and excited and exotic muons. Mass limits have been set for excited electrons of $756 \mathrm{GeV}$ for $\Lambda=1 \mathrm{TeV}$ in contact interaction models. For excited muons, mass limits up to $890 \mathrm{GeV}$ have been set, depending on assumptions 


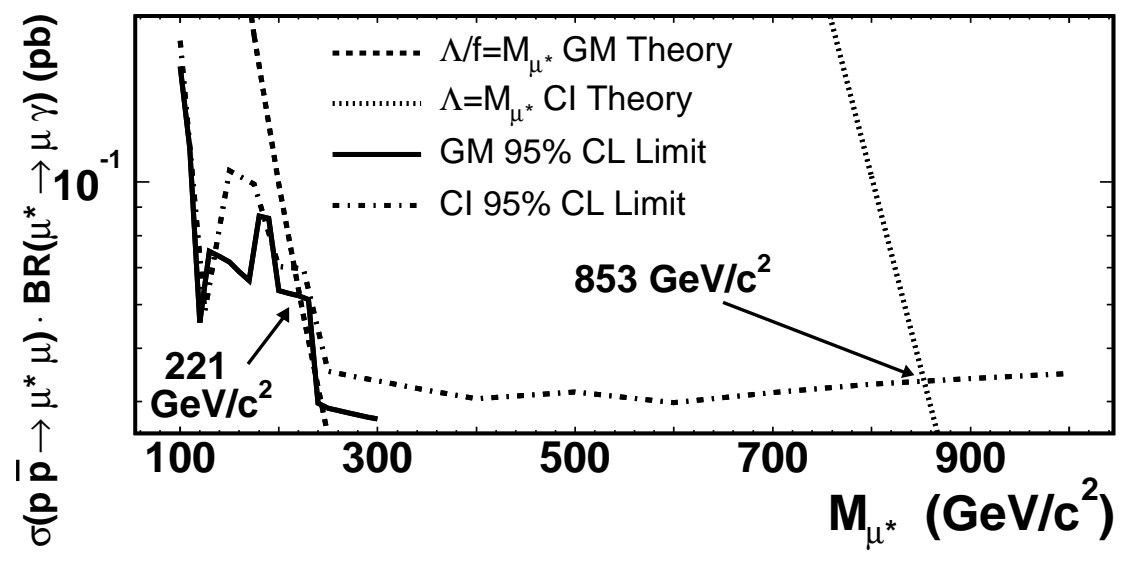

Figure 2: The CDF experimental cross section $\times$ branching ratio limits at 95\% C.L. for the CI (dashed-dotted line) and GM models (solid line), compared to the CI model prediction for $\Lambda=m_{\mu^{*}}$ (dotted line) and the GM model prediction for $\Lambda / f=m_{\mu^{*}}$ (dashed line). Also indicated are the mass values that are excluded by these data.

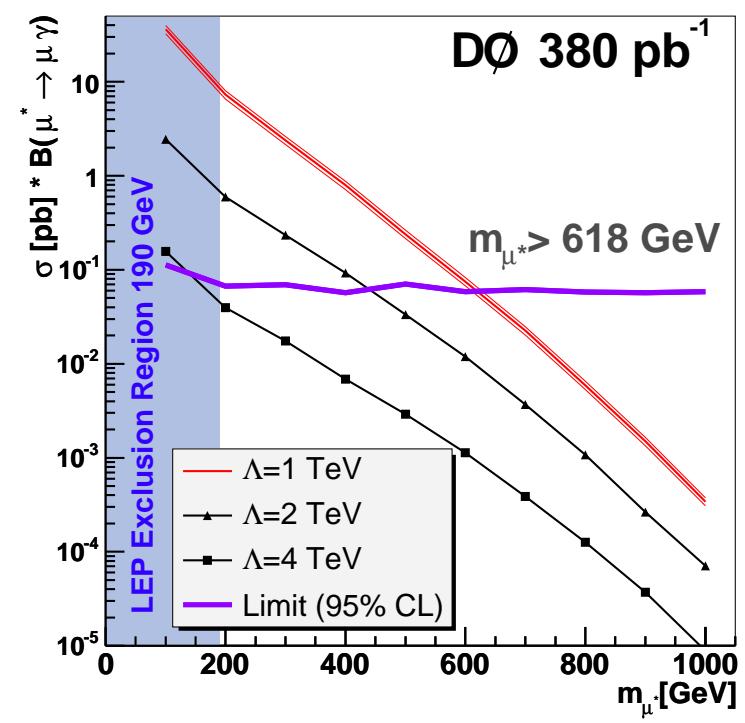

Figure 3: The $\mathrm{D} \varnothing$ measured cross section $\times$ branching fraction limit, compared to the contact interaction model prediction for different choices of $\Lambda$. For the case $\Lambda=1 \mathrm{TeV}$, the theoretical uncertainty of the model prediction is indicated. 
on $\Lambda$ and decay modes. For $\Lambda / f=m_{\mu^{*}}$, exotic muons in gauge-mediated models have been excluded for masses below $221 \mathrm{GeV}$.

\section{Bibliography}

\section{References}

[1] Slides: http://indico. cern. ch/contributionDisplay $\cdot$ py? contribId=106\&sessionId=15\&conf Id=24657

[2] H. Terazawa, M. Yasue, K. Akama and M. Hayashi, Phys. Lett. B 112, 387 (1982); F.M. Renard, Nuovo Cimento A77, 1 (1983); A. De Rujula, L. Maiani and R. Petronzio, Phys. Lett. B 140, 253 (1984); E.J. Eichten, K.D. Lane and M.E. Peskin, Phys. Rev. Lett. 50, 811 (1983).

[3] U. Baur, M. Spira and P.M. Zerwas, Phys. Rev. D 42, 815 (1990).

[4] K. Hagiwara, S. Komamiya, and D. Zeppenfeld, S. Phys. C 29, 115 (1985), and references therein; E. Boos et al., Phys. Rev. D 66, 013011 (2002), and references therein.

[5] D. Acosta et al. (CDF Collaboration), Phys. Rev. Lett. 94, 101802 (2005).

[6] V. M. Abazov et al. (DØ Collaboration), Phys. Rev. D 77, 091102(R) (2008).

[7] A. Abulencia et al. (CDF Collaboration), Phys. Rev. Lett. 97, 191802 (2006).

[8] V. M. Abazov et al. (DØ Collaboration), Phys. Rev. D 73, 111102(R) (2006). 